Article

\title{
In-Vitro Efficacy of Cefiderocol in \\ Carbapenem-Non-Susceptible Gram-Negative Bacilli of Different Genotypes in Sub-Region of North Rhine Westphalia, Germany
}

\author{
Beniam Ghebremedhin * and Parviz Ahmad-Nejad
}

Citation: Ghebremedhin, B.;

Ahmad-Nejad, P. In-Vitro Efficacy of Cefiderocol in Carbapenem-NonSusceptible Gram-Negative Bacilli of Different Genotypes in Sub-Region of North Rhine Westphalia, Germany. Pathogens 2021, 10, 1258. https:// doi.org/10.3390/pathogens10101258

Academic Editor: Sung-Pin Tseng

Received: 31 August 2021

Accepted: 27 September 2021

Published: 29 September 2021

Publisher's Note: MDPI stays neutral with regard to jurisdictional claims in published maps and institutional affiliations.

Copyright: (c) 2021 by the authors. Licensee MDPI, Basel, Switzerland. This article is an open access article distributed under the terms and conditions of the Creative Commons Attribution (CC BY) license (https:/ / creativecommons.org/licenses/by/ $4.0 /)$.
Institute of Medical Laboratory Diagnostics, Center for Clinical and Translational Research, Helios University Clinic Wuppertal, Witten/Herdecke University, Department of Health, 42283 Wuppertal, Germany; parviz.ahmad-nejad@helios-gesundheit.de

* Correspondence: beniam.ghebremedhin@uni-wh.de; Tel.: +49-(202)-896-2262

\begin{abstract}
In the last two decades, the worldwide dissemination of multidrug-resistant Gram-negative bacteria (MDR-GNB) has continued. Therapy options for such infections caused by MDR-GNB remain scarce, and only few new antimicrobial agents have been granted market approval. Cefiderocol has been approved for the treatment of infections associated with aerobic GNB with limited therapy options. This study evaluated the in vitro efficacy of cefiderocol against carbapenem-non-susceptible clinical GNB isolates from Germany. A total of 115 non-duplicate carbapenem-nonsusceptible GNB isolates, 61 (53.05\%) of which were Enterobacterales species and 54 (46.95\%) were non-fermenters (Acinetobacter baumanii and Pseudomonas aeruginosa), were investigated for their cefiderocol susceptibility. Minimum inhibitory concentrations (MICs) for cefiderocol were determined by disk diffusion, according to EUCAST (European committee for antimicrobial susceptibility testing). Susceptibility rates were based on EUCAST breakpoints. In the absence of a species-specific breakpoint, pharmacokinetic/-dynamic breakpoints were used. The most common pathogen was A. baumannii (33.91\%), followed by Klebsiella pneumoniae (31.3\%), P. aeruginosa (13.04\%) and Escherichia coli (9.57\%). Overall, $83.6 \%(51 / 61)$ of the Enterobacterales and $81.48 \%(44 / 54)$ of the non-fermenters were susceptible towards cefiderocol. In total, 20 species of Enterobacterales and non-fermenting GNB were resistant towards cefiderocol, irrespective of the isolation year (2014 to 2021). Moreover, the majority of the resistant isolates were among the OXA-23 producing A. baumannii $(n=7 / 26 ; 26.92 \%)$ from patients hospitalized during 2018 and 2019. Cefiderocol demonstrated high in vitro susceptibility rates against a wide range of carbapenem-non-susceptible GNB, including carbapenemase-producing isolates. Cefiderocol exhibited stability against hydrolysis by all carbapenemases, including metallo- $\beta$-lactamases (MBLs), except that few OXA-producing isolates exhibited resistance towards cefiderocol.
\end{abstract}

Keywords: cefiderocol; siderophore; Pseudomonas; Acinetobacter; Enterobacterales; antimicrobial resistance

\section{Introduction}

Infections due to multidrug-resistant Gram-negative bacteria (MDR-GNB), especially those associated with isolates which are non-susceptible towards carbapenem, have been very difficult to manage in the past two decades [1,2].

According to the World Health Organization (WHO), carbapenem-non-susceptible GNB (e.g., Escherichia coli, Klebsiella pneumoniae), including non-fermenters (e.g., Acinetobacter baumannii), are considered "critical" pathogens. The WHO declared the development of new antimicrobials against such MDR-GNB as a priority [3]; this is reasonable since there is an increasing number of MDR-GNB. This makes appropriate treatment difficult, resulting in high mortality rates [2,4-7]. According to the WHO, at least 700,000 people die each year due to MDR-related diseases, including 230,000 people who die due to MDR-associated tuberculosis [8]. Burnham et al. [9] re-estimated the deaths due to MDR-infections in the 
United States. In 2010, nearly two and a half million deaths occurred in the United States and approximately 70,000 of them died due to sepsis caused by MDR-GNB in US hospitals [9]. The authors conclude that their estimates for inpatient and outpatient deaths due to MDR-related infections gives more than 150,000 deaths, which is almost 7-fold higher than that reported by the Centers for Disease Control and Prevention (CDC: 23,000 deaths) in 2010 [9]. For the United States, the CDC reports that more than 35,000 people die out of $>2.8$ million MDR-related infections each year [10]. The European Centre for Disease Prevention and Control (ECDC) states on its website that 33,000 people die in Europe due to an infection associated with antibiotic-resistant bacteria each year [11]. This report coincides with a study by Cassini et al. [12]. According to Cassini et al. [12] MDR-GNB are responsible for approximately 33,000 deaths in Europe annually. However, there is an urgent need for better global surveillance and reporting mechanisms and databases for MDR-related infections and mortality to avoid or at least minimize controversies.

Among the recent antimicrobial agents, cefiderocol is the first siderophore cephalosporin which has been approved by the Food and Drug Administration (FDA) and European Medicines Agency (EMA), against different species of Enterbacterales and non-fermenting Gram-negative bacteria with different carbapenemase-types/sub-types that may have the potential to fill some of the remaining gaps in the treatment of MDR-GNB-associated infections [13-17].

The chlorocatechol side chain allows cefiderocol to bind extracellular free iron and to form $\mathrm{Fe}^{3+}$ chelate complex. This complex is formed by bacteria recognized as a siderophore and using active bacterial iron transport mechanisms as a "Trojan horse" through the outer membrane of GNB into the periplasmic space where it then binds to the penicillin-binding protein 3 (PBP-3) and thus inhibits the synthesis of peptidoglycan bacterial cell wall which leads to lysis and death of the bacteria [13].

Cefiderocol shows stability against numerous $\beta$-lactamases of Ambler classes A to $\mathrm{D}$, including carbapenemases and thus exhibits activity against Enterobacterales, Pseudomonas aeruginosa, the A. baumannii complex, but also against some other GNBs [13,14]. There is no effectiveness against Gram-positive pathogens (such as staphylococci, streptococci or enterococci), anaerobes and intracellular bacteria, e.g., Mycoplasma pneumoniae [15]. Additionally, and similar to the oxyimino- $\beta$-lactams, ceftazidime and cefepime, the stability of cefiderocol to $\beta$-lactamases and activity against GNB, including P. aeruginosa, is enhanced via the pyrrolidinium group on the C3 side chain and the carboxypropyloxyimino group on the $\mathrm{C} 7$ side chain, which also enhances cefiderocols's stability towards $\beta$-lactamases [16]. Cefiderocol appears to be well positioned to cover the increasing number of infections caused by carbapenem-resistant and MDR-GNB, including ESBL- and carbapenemase-producing isolates [15]. Hence, we investigated different carbapenem-nonsusceptible MDR-GNB Enterobacterales species and non-fermenting GNB, P. aeruginosa, and A. baumannii complex for their susceptibility towards cefiderocol.

\section{Results}

The cefiderocol MIC levels were highly variable depending on the GNB species, with an overall MIC range of $\leq 0.03$ to $24 \mathrm{mg} / \mathrm{L}$. The species E. coli, C. freundii, and S. marcescens uniformly displayed $100 \%$ susceptibility to cefiderocol, regardless of the harbored carbapenemase genes (Table 1). When the EUCAST non-species-specific pharmacokineticpharmacodynamic (PK-PD) breakpoints were applied for A. baumannii, both A. baumannii and K. pneumoniae had resistance rates of $23.1 \%$ and $19.4 \%$, respectively, depending on the resistance genes they harbored. In contrast to A. baumannii, only $6.7 \%$ of the P. aeruginosa isolates were resistant towards cefiderocol. 


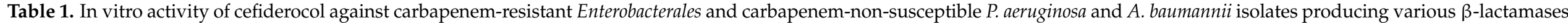
(total $n=115$ ).

\begin{tabular}{|c|c|c|c|c|c|c|c|c|c|c|c|c|c|c|c|c|c|c|c|c|c|c|c|c|c|}
\hline \multicolumn{26}{|c|}{ MIC in $\mathrm{mg} / \mathrm{L}$} \\
\hline GNB Species & 0.016 & 0.023 & 0.032 & 0.047 & 0.064 & 0.094 & 0.125 & 0.19 & 0.25 & 0.38 & 0.39 & 0.5 & 0.75 & 1 & 1.5 & 2 & 3 & 4 & 8 & 9 & 12 & 16 & 17 & 24 & Total (Species) \\
\hline A. baumannii & 2 & & & & & 1 & 1 & 5 & 2 & 5 & 1 & 2 & 3 & 3 & 3 & 2 & 1 & 2 & 1 & & 2 & 2 & & 1 & 39 \\
\hline C. freundii & 1 & & & & & & & & & & & & & & 1 & 1 & & & & & & & & & 3 \\
\hline E. cloacae & & & & & 1 & & & & & & & & & & 2 & & & & & 1 & & & & & 4 \\
\hline E. coli & 4 & & & & & & 1 & & 1 & 2 & & & & 1 & 1 & 1 & & & & & & & & & 11 \\
\hline K. aerogenes & & & 1 & & & & & & & & & & & & & & & & & & & 1 & & & 2 \\
\hline K. oxytoca & 1 & & & & & & & & & & & & & & & & & 1 & & & & & & & 2 \\
\hline K. pneumoniae & 4 & 5 & 2 & 4 & 2 & & 3 & & 2 & 2 & & 3 & & & & 2 & 4 & & & & & 2 & 1 & & 36 \\
\hline P. aeruginosa & & & & & & & & 2 & 2 & & & 3 & 1 & 1 & 1 & 4 & 1 & & & & & & & & 15 \\
\hline S. marcescens & 1 & & & & & & & & 1 & & & & & & & & & & & & & & & & 2 \\
\hline total (isolates) & 14 & 5 & 3 & 4 & 3 & 1 & 5 & 7 & 8 & 9 & 1 & 8 & 4 & 5 & 8 & 10 & 3 & 3 & 1 & 1 & 2 & 5 & 1 & 1 & 115 \\
\hline
\end{tabular}




\subsection{Enterobacterales}

The mean susceptibility rate of all Enterobacterales species towards cefiderocol was approximately $81 \%$. Among the surveillance isolates gathered in 2014-2021 (Table 1), all E. coli isolates $(n=11)$ had cefiderocol MICs $\leq 2 \mathrm{mg} / \mathrm{L}$. The mean cefiderocol MIC was $0.25 \mathrm{mg} / \mathrm{L}$. This applies also for the two S. marcenscens isolates with cefiderocol MICs of 0.016 and $0.25 \mathrm{mg} / \mathrm{L}$, and the single $R$. ornithinolytica isolate with an MIC value of $0.016 \mathrm{mg} / \mathrm{L}$, respectively. Similarly, all three C. freundii isolates had cefiderocol MICs $\leq 2 \mathrm{mg} / \mathrm{L}$. The same is true for three E. cloacae isolates $(n=3 / 4)$ with MICs $\leq 2 \mathrm{mg} / \mathrm{L}$, exlusively one E. cloacae ( $n=1 / 4)$ which was resistant towards cefiderocol with MIC value of $9 \mathrm{mg} / \mathrm{L}$. This isolate originated from a clinical sample in 2021 and co-carried VIM-1 and OXA-48 genes. We identified a single Klebsiella aerogenes isolate with a relatively low cefiderocol MIC value of $0.064 \mathrm{mg} / \mathrm{L}$. The second K. aerogenes isolate (isolation year 2019) was inhibited at cefiderocol MIC $=16 \mathrm{mg} / \mathrm{L}$ without carrying any carbapenemase gene, but AmpC gene.

As for the 36 investigated K. pneumoniae isolates, 3 of $5 \mathrm{KPC}-2(n=3 / 5)$ and all $4 \mathrm{KPC}$ $3(n=4 / 4)$ producing isolates were cefiderocol-susceptible, with MIC values ranging from 0.023 and $2 \mathrm{mg} / \mathrm{L}$ and 0.064 and $2 \mathrm{mg} / \mathrm{L}$, respectively. All OXA-48 producing K. pneumoniae isolates $(n=12 / 12)$ were cefiderocol-susceptible with MIC values $\leq 0.5 \mathrm{mg} / \mathrm{L}$. Few K. pneumoniae isolates harboring the carbapenemase genes OXA-181 $(n=2 / 2)$, OXA-204 $(n=1 / 1)$, OXA-232 $(n=1 / 1)$, VIM-1 $(n=1 / 1)$, and VIM-2 $(n=1 / 1)$ uniformly displayed $100 \%$ susceptibility towards cefiderocol with MIC values $\leq 0.032$.

However, the overall susceptibility rate was $80.6 \%$ for all the K. pneumoniae isolates irrespective of their resistance bla genes, defining this group as the most cefiderocol-resistant within the Enterobacterales.

\subsection{P. aeruginosa and A. baumannii}

There were 15 isolates of P. aeruginosa gathered from 2014 to 2021 (Table 1), and 93.3\% had cefiderocol MIC $\leq 2 \mathrm{mg} / \mathrm{L}$. The $\beta$-lactamase gene detected within these isolates was bla $a_{\text {VIM-2 }} \beta$-lactamase $(n=9 / 15)$, all were cefiderocol-susceptible within the MIC range of 0.19 and $2 \mathrm{mg} / \mathrm{L}$.

There were 39 isolates of A. baumannii gathered from 2014 to 2021 , and $77.9 \%$ had cefiderocol MICs $\leq 2 \mathrm{mg} / \mathrm{L}$. When the EUCAST non-species-specific pharmacokineticpharmacodynamic (PK-PD) breakpoints were applied for A. baumannii, 23.1\% of the carbapenem-resistant $A$. baumannii isolates exhibited resistance towards cefiderocol with MIC $>2$ to $16 \mathrm{mg} / \mathrm{L}$, especially those carrying the $b a_{\mathrm{OXA}}$-23-type $\beta$-lactamase. Among the isolates harboring this gene $29.1 \%(n=7 / 24)$ were resistant towards cefiderocol with MIC values ranging from 3 to $24 \mathrm{mg} / \mathrm{L}$ (Table 2).

Table 1 summarizes the in vitro activity of cefiderocol against carbapenemase-producing isolates and carbapenemase-negative isolates of carbapenem-non-susceptible Enterobacterales and carbapenem-non-susceptible P. aeruginosa and A. baumannii.

\subsection{Cefiderocol-Susceptible Enterobacterales, P. aeruginosa and A. baumannii with Emphasis on bla $a_{O X A-23-t y p e}$ and bla $a_{O X A-48-t y p e} \beta$-lactamase}

To emphasize the high MIC levels for cefiderecol in particular isolates among the susceptible GNB species with various carbapenemase genes, we selected the predominant species with their harbored genes in Table 3 . This table indicates that $A$. baumannii isolates carrying the $b l a$ OXA-23-type $\beta$-lactamase show a tendency for higher MIC levels $>0.125$ to $2 \mathrm{mg} / \mathrm{L}$, with the emphasis of MIC frequency of 0.19 . Whereas the K. pneumoniae isolates carrying the $b l a_{\mathrm{OXA}}-48$-type $\beta$-lactamase displayed frequently lower MIC levels for cefiderocol: $n=7$ of 12 isolates with MIC levels $0.023 \mathrm{mg} / \mathrm{L}$ and $0.047 \mathrm{mg} / \mathrm{L}$. The same applies for $E$. coli isolates carrying the $b l_{\text {OXA-48-type }} \beta$-lactamase, at least for $n=3 / 3$ isolates with $\mathrm{MIC}=0.016$ and $0.125 \mathrm{mg} / \mathrm{L}$ ). 
Table 2. A subset of cefiderocol-resistant and carbapenem-non-susceptible Enterobacterales, P. aeruginosa and A. baumannii isolates producing various $\beta$-lactamases, $n=20 ; \mathrm{R} \%$, resistance rate in $\%$.

\begin{tabular}{|c|c|c|c|c|c|c|c|c|c|c|}
\hline \multicolumn{11}{|c|}{ MIC mg/L Cefiderocol } \\
\hline & 3 & 4 & 8 & 9 & 12 & 16 & 17 & 24 & & \\
\hline GNB Species & & & & & & & & & $n$ & $\mathbf{R} \%$ \\
\hline A. baumannii & 1 & 2 & 1 & & 2 & 2 & & 1 & 9 & $23.1 \%$ \\
\hline$N D M-1 /-6+O X A-23$ & & 1 & & & & & & & 1 & \\
\hline NDM-9 & & & & & & 1 & & & 1 & \\
\hline$O X A-23$ & 1 & 1 & 1 & & 2 & 1 & & 1 & 7 & \\
\hline E. cloacae & & & & 1 & & & & & 1 & $25.0 \%$ \\
\hline VIM-1/OXA-48 & & & & 1 & & & & & 1 & \\
\hline K. aerogenes & & & & & & 1 & & & 1 & $50.0 \%$ \\
\hline AmpC & & & & & & 1 & & & 1 & \\
\hline K. oxytoca & & 1 & & & & & & & 1 & $50.0 \%$ \\
\hline VIM-4 & & 1 & & & & & & & 1 & \\
\hline К. pneumoniae & 4 & & & & & 2 & 1 & & 7 & $19.4 \%$ \\
\hline ESBL & 2 & & & & & & & & 2 & \\
\hline$K P C-2$ & 1 & & & & & 1 & & & 2 & \\
\hline $\begin{array}{c}\text { NDM-1/NDM-6/NDM- } \\
\text { 16/OXA-48 }\end{array}$ & 1 & & & & & & & & 1 & \\
\hline$N D M-5 /-20$ & & & & & & & 1 & & 1 & \\
\hline NDM-7/NDM-19 & & & & & & 1 & & & 1 & \\
\hline P. aeruginosa & 1 & & & & & & & & 1 & $6.7 \%$ \\
\hline AmpC & 1 & & & & & & & & 1 & \\
\hline total & 6 & 3 & 1 & 1 & 2 & 5 & 1 & 1 & 20 & \\
\hline
\end{tabular}

Table 3. A selection of cefiderocol-susceptible and carbapenem-non-susceptible Enterobacterales, P. aeruginosa and A. baumannii isolates producing various $\beta$-lactamases-with emphasis on bla OXA-23-type and bla OXA-48-type $\beta$-lactamase, $n=84$.

\begin{tabular}{|c|c|c|c|c|c|c|c|c|c|c|c|c|c|c|c|c|c|}
\hline \multirow[b]{2}{*}{ GNB Species, Genes } & \multicolumn{17}{|c|}{ MIC mg/L Cefiderocol } \\
\hline & 0.016 & 0.023 & 0.032 & 0.047 & 0.064 & 0.094 & 0.125 & 0.19 & 0.25 & 0.38 & 0.39 & 0.5 & 0.75 & 1.0 & 1.5 & 2.0 & $n$ \\
\hline A. baumannii & 2 & & & & & 1 & 1 & 5 & 2 & 5 & 1 & 2 & 3 & 3 & 3 & 2 & 30 \\
\hline GIM-1 & & & & & & & & & & & & & & 1 & & & 1 \\
\hline$N D M-1 /-6$ & 1 & & & & & & & & & 1 & & & & & & & 2 \\
\hline$N D M-1 /-6+O X A-23$ & & & & & & & & & & & & & & & & 1 & 1 \\
\hline NDM-2 & & & & & & & & & & & & & & & 1 & & 1 \\
\hline$O X A-164$ & & & & & & 1 & & & & & & & & & & & 1 \\
\hline$O X A-23$ & & & & & & & 1 & 5 & 1 & 2 & 1 & 2 & 2 & 2 & 1 & & 17 \\
\hline$O X A-40$ & 1 & & & & & & & & & & & & & & & & 1 \\
\hline OXA-58 & & & & & & & & & & & & & 1 & & & 1 & 2 \\
\hline$O X A-72$ & & & & & & & & & 1 & 2 & & & & & 1 & & 4 \\
\hline E. coli & 4 & & & & & & 1 & & 1 & 2 & & & & 1 & 1 & 1 & 11 \\
\hline KPC-2 & & & & & & & & & & 1 & & & & & & & 1 \\
\hline$K P C-3$ & 2 & & & & & & & & & & & & & & & & 2 \\
\hline NDM-3 & & & & & & & & & 1 & & & & & & & & 1 \\
\hline NDM-5 & & & & & & & & & & & & & & & 1 & 1 & 2 \\
\hline$O X A-181$ & & & & & & & & & & 1 & & & & 1 & & & 2 \\
\hline$O X A-48$ & 2 & & & & & & 1 & & & & & & & & & & 3 \\
\hline K. pneumoniae & 4 & 5 & 2 & 4 & 2 & & 3 & & 2 & 2 & & 3 & & & & 2 & 29 \\
\hline ESBL + other mechanisms & & & & & & & & & & & & & & & & 1 & 1 \\
\hline$K P C-2$ & & 1 & & 1 & & & & & & 1 & & & & & & & 3 \\
\hline$K P C-3$ & & & & & 1 & & & & 1 & & & 1 & & & & 1 & 4 \\
\hline$N D M-1 /-6$ & 1 & & & & & & & & & & & & & & & & 1 \\
\hline NDM-1/NDM-6/NDM-16 & & & & & & & & & & & & 1 & & & & & 1 \\
\hline OXA-181 & & & 1 & & & & & & 1 & & & & & & & & 2 \\
\hline$O X A-181 / O X A-48$ & & & & & 1 & & & & & & & & & & & & $\overline{1}$ \\
\hline OXA-204 & 1 & & & & & & & & & & & & & & & & 1 \\
\hline$O X A-232$ & & & & & & & & & & 1 & & & & & & & 1 \\
\hline$O X A-48$ & 2 & 4 & 1 & 3 & & & 1 & & & & & 1 & & & & & 12 \\
\hline VIM-1 & & & & & & & 1 & & & & & & & & & & 1 \\
\hline VIM-2 & & & & & & & 1 & & & & & & & & & & 1 \\
\hline P. aeruginosa & & & & & & & & 2 & 2 & & & 3 & 1 & 1 & 1 & 4 & 14 \\
\hline AmpC + other mechanisms & & & & & & & & & & & & 1 & & 1 & 1 & 2 & 5 \\
\hline VIM-2 & & & & & & & & 2 & 2 & & & 2 & 1 & & & 2 & 9 \\
\hline total isolates & 10 & 5 & 2 & 4 & 2 & 1 & 5 & 7 & 7 & 9 & 1 & 8 & 4 & 5 & 5 & 9 & 84 \\
\hline
\end{tabular}




\section{Discussion}

Cefiderocol, an innovative parenteral siderophore cephalosporin, has demonstrated potent bactericidal efficacy against Gram-negative bacilli (GNB) such as carbapenem-nonsusceptible Enterobacterales species, Pseudomonas aeruginosa, and Acinetobacter baumannii that are multidrug-resistant (MDR) and producers of diverse carbapenemases and other $\beta$-lactamases.

Its efficacy has been investigated in large international surveillance studies carried out since 2014 (SIDERO-WT studies), covering over 28,000 Gram-negative bacteria. Overall, the $\mathrm{MIC}_{90}$ for Enterobacterales (including Escherichia coli, Klebsiella spp., Citrobacter spp., Enterobacter spp., Serratia spp. and other species) ranged from 0.25 to $1 \mathrm{mg} / \mathrm{L}$, with no significant geographical or temporal differences. The overall activity of cefiderocol against Enterobacterales from surveillance studies revealed that $>98 \%$ of isolates were inhibited at concentrations of $2 \mathrm{mg} / \mathrm{L}$. However, this was retained against most isolates resistant to expanded-spectrum cephalosporins and carbapenems, including those producing different types of serine carbapenemases and metallo- $\beta$-lactamases. Naas et al. [17] evaluated the in vitro activity of cefiderocol and comparators against Gram-negative bacilli (GNB) clinical isolates from France. Cefiderocol demonstrated high in vitro susceptibility rates against a wide range of GNB, including meropenem-resistant strains, and was significantly more active than comparators against pneumonia isolates. Overall, 99.0\% Enterobacterales and $(99.7 \%)$ non-fermenters were cefiderocol susceptible, including $100 \%$ of meropenemresistant S. maltophilia and P. aeruginosa isolates. In contrast to the findings of Naas et al. [17], the cefiderocol susceptibility rate of our isolates was lower for both GNB groups, the Enterobacterales and the non-fermenters. Our results are consistent with previous reports by Mushtaq et al. [18], at least for Enterobacterales and A. baumannii. The overall efficacy rates of cefiderocol in our carbapenem-non-susceptible GNB isolates were: $81.9 \%$ for Enterobacterales species, $93.3 \%$ P. aeruginosa, and the lowest rate of $76.9 \%$ for A. baumannii.

Mushtaq et al. [18] evaluated cefiderocol MICs against GNB by use of iron-depleted Mueller-Hinton broth, the gold standard method. The panel comprised 305 isolates of Enterobacterales, 111 of P. aeruginosa, and 99 of A. baumannii, and all were carbapenemnon-susceptible and MDR to other agents. Of all Enterobacterales, $78.7 \%$ were inhibited at $2 \mathrm{mg} / \mathrm{L}$ cefiderocol, with rates of 80 to $100 \%$ for isolates with all modes of carbapenem resistance except NDM enzymes (41.0\% inhibited at $2 \mathrm{mg} / \mathrm{L}$ ) or combinations of ESBL and other mechanisms (61.5\% inhibited at $2 \mathrm{mg} / \mathrm{L})$. Cefiderocol inhibited $81.1 \%$ of all P. aeruginosa isolates at $2 \mathrm{mg} / \mathrm{L}$, with rates of 80 to $100 \%$ for isolates with VIM, IMP, GES, or VEB $\beta$-lactamases and slightly lower rates for those with NDM $(45.5 \%$ at $2 \mathrm{mg} / \mathrm{L})$ and PER $(66.7 \%$ at $2 \mathrm{mg} / \mathrm{L})$ enzymes. $80.8 \%$ of the $A$. baumannii isolates were inhibited at $2 \mathrm{mg} / \mathrm{L}$, with rates of $>85 \%$ for isolates with OXA-51-like, $-23,-24$, or -58 enzymes and $50 \%$ at $2 \mathrm{mg} / \mathrm{L}$ for those with NDM carbapenemases. In our study, $29.2 \%$ of the OXA-23 producing A. baumannii isolates were resistant towards cefiderocol at MIC $>2$ to $24 \mathrm{mg} / \mathrm{L}$ or at $<17 \mathrm{~mm}$ of inhibition zone diameter. However, Malik et al. [19] examined the resistance mechanisms in 12 A. baumannii isolates with cefiderocol MICs ranging from $\leq 0.03$ to $32 \mathrm{mg} / \mathrm{L}$. Their resistance to this new agent could not be explained by any $\beta$-lactamase activity.

Iregui et al. [20] determined higher cefiderocol MICs in endemic E. coli and K. pneumoniae isolates at the Medical Centers in New York City that were cephalosporin resistant. The activity of cefiderocol has been reported to be maintained in Enterobacterales possessing a wide variety of carbapenemases, including KPC, NDM VIM, IMP, and OXA-48. Of the 78 carbapenem-resistant $A$. baumannii isolates, including 47 with OXA-23-type $\beta$-lactamases, the $\mathrm{MIC}_{90}$ value was $8 \mathrm{mg} / \mathrm{L}$, which was identical to the subset of isolates with OXA-23type $\beta$-lactamases. Moreover, Malik et al. [19] conclude that the cefiderocol resistance in A. baumannii isolates was associated with reduced expression of the siderophore receptor gene pirA. Mutations involving PBP3 may have contributed to resistance in one isolate. Future studies should investigate the role of the siderophore receptors. Poirel et al. [21] investigated a series of A. baumannii clinical isolates with elevated MICs of cefiderocol 
and showed that PER-like $\beta$-lactamases, and to a lesser extent NDM-like $\beta$-lactamases, significantly contributed to reduced susceptibility towards cefiderocol.

Furthermore, Jacobs et al. [22] revealed higher MICs for cefiderocol in Enterobacterales possessing an ESBL, carbapenemase, or AmpC-type $\beta$-lactamase compared with isolates lacking these enzymes.

Moreover, Ito et al. and Ito-Horiyama et al. [23,24] investigated the mechanism of action of cefiderocol has revealed that the specific outer membrane iron transporters CirA and Fiu in E. coli and PiuA in P. aeruginosa are involved in the active transport of cefiderocol. Such mutations in GNB resulted in deficiency in the activity of these transporter molecules and revealed significantly $\geq 16$-fold increased MICs of cefiderocol. However, experiments with purified enzyme extracts of frequent carbapenemases, e.g., KPC-3, IMP-1, VIM-2, NDM-1, OXA-48, OXA-23 $\beta$-lactamases, revealed that cefiderocol remained stable when exposed to these enzymes [23-25].

In this study, we found that NDM-1/-6 positive isolates ( $n=4$ of $4,100 \%$ ) were inhibited by $\leq 2 \mathrm{mg} / \mathrm{L}$ cefiderocol. However, this was not true for such isolates which were positive for NDM-5/-20, NDM-7/NDM-19, and NDM-9. These carbapenemase genes encoding for New Delhi metallo- $\beta$-lactamase were harbored by A. baumannii $(n=1)$ and K. pneumoniae isolates $(n=2)$ exhibiting MIC levels $>16 \mathrm{mg} / \mathrm{L}$ of cefiderocol. The isolates were gained from clinical specimens during 2014 to 2017. Furthermore, Kazmierczak et al. [15] found that $35.7 \%$ of the NDM-positive isolates were not inhibited by $\leq 4 \mathrm{mg} / \mathrm{L}$ cefiderocol. The authors received these K. pneumoniae isolates from Turkey with a cefiderocol MIC level of $8 \mathrm{mg} / \mathrm{L}$ that carried NDM-1 and a CTX-M-1-type ESBL. According to their sequence analysis of the porin gene, one isolate had lost OmpK35 and three others were expected to produce truncated OmpK36 proteins of different lengths. These are findings which underscore the regional or intercontinental diversity of strains with non-susceptibility towards cefiderocol.

The previously approved antimicrobial agents are characterized by a good effect on MDR-GNB. Resistance to these antimicrobials is currently still rare, but has already emerged partially before their approval, e.g., cefiderocol, as observed in the bacterial population of the current study. Due to their very sufficient efficacy towards various MDR-GNB, these antimicrobials should not be used as new broad-spectrum antibiotics in calculated therapy-despite the broad spectrum of activity against MDR-GNB. The current study examined the activity of cefiderocol, a promising parenteral siderophore cephalosporin with time-dependent bactericidal activity against GNB, especially those in late-stage clinical development for treatment of infections caused by MDR-GNB. The distribution of carbapenemase types varies among isolates in our region and other regions, or even continental regions, which underscores the importance of knowing the local incidence of different resistance mechanisms when evaluating treatment options. Regardless of GNB species, cefiderocol strongly demonstrated sufficient in vitro activity towards carbapenemase-producing isolates. Cefiderocol represents an addition to the limited last resort of existing antimicrobial agents available for treatment of severe infections caused by MDR-GNB.

We believe, due to the heterogeneity of carbapenem-non-susceptible MDR-GNB, that in order to maintain the effectiveness of this reserve antimicrobial agent for as long as possible, it should only be administered if an additional benefit compared to established agents has been proven, e.g., via antimicrobial susceptibility testing. The therapy options for infections with MDR-GNB should be standardized within the local clinic in order to avoid inefficient therapies as best as possible.

This study included a relatively small number of isolates, particularly for any individual species. Furthermore, isolates were derived from a single region, which may limit the generalizability of our findings to other regions with differences in the composition of common Gram-negative resistance mechanisms. Though our region is a rather lowprevalence area for carbapenemase-producing GNB, the current results need to be verified 
in a larger study that includes geographically diverse isolates with higher frequency of different bla genes.

\section{Materials and Methods}

One-hundred fifteen non-duplicate clinical GNB isolates of the years 2014 to 2021 (Table 4) were identified using matrix-assisted laser-desorption ionization time-of-flight mass spectrometry (MALDI-TOF-MS; Bruker Daltonics, Bremen, Germany). AST results were determined using the BD Phoenix automated system (BD Diagnostics, Heidelberg, Germany).

Table 4. The bacterial population which was investigated for cefiderocol susceptibility. A. baumannii, E. coli, and K. pneumoniae producing various $\beta$-lactamases and other resistance mechanisms were the predominant species among the total number of the isolates, $n=115$.

\begin{tabular}{|c|c|c|}
\hline GNB Species, Harbored Genes & $n$ & $\%$ of Total Isolates \\
\hline A. baumannii & 39 & $33.91 \%$ \\
\hline GIM-1 & 1 & \\
\hline$N D M-1 /-6$ & 2 & \\
\hline$N D M-1 /-6+O X A-23$ & 2 & \\
\hline$N D M-2$ & 1 & \\
\hline NDM-9 & 1 & \\
\hline$O X A-164$ & 1 & \\
\hline$O X A-23$ & 24 & \\
\hline$O X A-40$ & 1 & \\
\hline$O X A-58$ & 2 & \\
\hline OXA-72 & 4 & \\
\hline C. freundii & 3 & $2.61 \%$ \\
\hline$A m p C$ & 1 & \\
\hline$O X A-162$ & 1 & \\
\hline VIM-1 & 1 & \\
\hline E. cloacae & 4 & $3.48 \%$ \\
\hline$N D M-1 /-6$ & 1 & \\
\hline VIM-1 & 1 & \\
\hline$V I M-1 / O X A-48$ & 1 & \\
\hline VIM-4 & 1 & \\
\hline E. coli & 11 & $9.57 \%$ \\
\hline$K P C-2$ & 1 & \\
\hline$K P C-3$ & 2 & \\
\hline NDM-3 & 1 & \\
\hline NDM-5 & 2 & \\
\hline$O X A-181$ & 2 & \\
\hline$O X A-48$ & 3 & \\
\hline K. aerogenes & 2 & $1.74 \%$ \\
\hline$A m p C$ & 1 & \\
\hline$O X A-48$ & 1 & \\
\hline K. oxytoca & 2 & $1.74 \%$ \\
\hline$K P C-2$ & 1 & \\
\hline VIM-4 & 1 & \\
\hline K. pneumoniae & 36 & $31.30 \%$ \\
\hline$E S B L$ & 3 & \\
\hline$K P C-2$ & 5 & \\
\hline$K P C-3$ & 4 & \\
\hline NDM-1/-6 & 1 & \\
\hline NDM-1/NDM-6/NDM-16 & 1 & \\
\hline NDM-1/NDM-6/NDM-16/OXA-48 & 1 & \\
\hline NDM-5/-20 & 1 & \\
\hline$N D M-7 / N D M-19$ & 1 & \\
\hline OXA-181 & 2 & \\
\hline OXA-181/OXA-48 & 1 & \\
\hline
\end{tabular}


Table 4. Cont.

\begin{tabular}{ccc}
\hline GNB Species, Harbored Genes & $n$ & \% of Total Isolates \\
\hline OXA-204 & 1 & \\
OXA-232 & 1 & \\
OXA-48 & 12 & \\
VIM-1 & 1 & \\
VIM-2 & 1 & $13.04 \%$ \\
P. aeruginosa & 15 & \\
\hline AmpC & 6 & $0.87 \%$ \\
VIM-2 & 9 & $1.74 \%$ \\
R. ornithinolytica & 1 & \\
KPC-2 & 1 & \\
\hline S. marcescens & 2 & \\
\hline OXA-48 & 1 & \\
\hline total & 1 & \\
\hline
\end{tabular}

These isolates had been collected in various surveillance studies performed in our Helios hospitals in North Rhine Westphalia between 2014 and 2021 and either characterized in our molecular laboratory or were sent to the German National Reference Centre for Multidrug-Resistant Gram-negative Bacteria for molecular typing Reference strains for verification. Escherichia coli DSM 1103 and Pseudomonas aeruginosa DSM 1117 were used for quality control. For all isolates with resistance to third-generation cephalosporins and/or carbapenems, the presence of beta-lactamase genes was tested via Real-Time$\mathrm{PCR} /$ sequencing, as previously described [26-31].

Antimicrobial susceptibility testing (AST) was interpreted according to EUCAST clinical breakpoints version 11.0 EUCAST, www.eucast.org (accessed on 31 August 2021), (Table 5), [31]. AST for cefiderocol was determined by epsilon test (Bestbion/Liofilchem, Cologne, Germany) on Mueller-Hinton agar according to the manufacturer's protocol. From an overnight agar plate, well-isolated colonies were suspended in saline to achieve a $0.5 \mathrm{McF}$ arland standard turbidity. By use of a sterile cotton swab, the inoculum was streaked over the entire area of the Mueller-Hinton (MH) agar plate. Thereafter, the test strip was firmly applied to the surface of the inoculated agar plate and incubated at $35 \pm 1{ }^{\circ} \mathrm{C}$ for $16-20 \mathrm{~h}$. According to the manufacturer's instruction for reading the plates after the required incubation period, and only when an even lawn of growth was distinctly visible, the MIC value was read where the relevant inhibition ellipse intersects the strip. For bactericidal antimicrobials like cefiderocol, the MIC endpoint should be read at complete inhibition of growth. Haze and macro- or micro-colonies within $3 \mathrm{~mm}$ from the strip should be read as growth (Technical sheet from Bestbion/Liofilchem, Cologne, Germany).

Table 5. EUCAST has values of $S \leq 2 \mathrm{mg} / \mathrm{L}$ and $\mathrm{R}>2 \mathrm{mg}$ /L for Enterobacterales and P. aeruginosa-EUCAST, European Committee on Antimicrobial Susceptibility Testing; S, susceptible; R, resistant. §: Non-speciesspecific pharmacokinetic-pharmacodynamic (PK-PD) breakpoint. \#: EUCAST provided disk correlates associated with the susceptible PK-PD breakpoint for A. baumannii.

\begin{tabular}{ccccc}
\hline \multirow{2}{*}{ Gram-Negative Bacilli } & \multicolumn{2}{c}{ MIC (mg/L) } & \multicolumn{2}{c}{ Inhibition Zone Diameter (mm) } \\
\cline { 2 - 5 } & $\mathbf{S}$ & $\mathbf{R}$ & $\mathbf{S}$ & $\mathbf{R}$ \\
\hline Enterobacterales spp. & $\leq 2$ & $>2$ & $\geq 22$ & $<22$ \\
Pseudomonas aeruginosa & $\leq 2$ & $>2$ & $\geq 22$ & $<22$ \\
Acinetobacter baumannii & $\leq 2 \S$ & $>2 \S$ & $\geq 17^{\#}$ & \\
\hline
\end{tabular}

Growth along the entire gradient i.e., no inhibition ellipse, indicates that the value is greater than or equal to $(\geq)$ the highest value on the scale. An inhibition ellipse that intersects below the lower end of the scale is read as less than $(<)$ the lowest value. Intersection between two scale segments should be rounded up to the higher value. An MIC of 
$0.125 \mathrm{mg} / \mathrm{L}$ is considered the same as $0.12 \mathrm{mg} / \mathrm{L}$ for reporting purposes (Technical sheet from Bestbion/Liofilchem, Cologne, Germany).

The European Committee on Antimicrobial Susceptibility Testing (EUCAST) has set cefiderocol breakpoints with MIC values of $\leq 2 \mathrm{mg} / \mathrm{L}$ and $>2 \mathrm{mg} / \mathrm{L}$ for susceptible and resistant categories, respectively, for Enterobacterales and Pseudomonas spp., and also pharmacokinetic/pharmacodynamic (PK/PD) breakpoint MIC values of $\leq 2 \mathrm{mg} / \mathrm{L}$ for susceptibility, while a breakpoint was not set for A. baumannii due to insufficient clinical evidence. However, A. baumannii was susceptible if inhibition zone diameter for cefiderocol disk was $>17 \mathrm{~mm}$ [31].

Author Contributions: Conceptualization, B.G. and P.A.-N.; Data Curation, B.G. and P.A.-N.; Project Supervision, B.G.; Validation, B.G.; Writing-Original Draft Preparation, B.G.; Writing-Review \& Editing, B.G. and P.A.-N. All authors have read and agreed to the published version of the manuscript.

Funding: This research is funded by the by the German Society of Clinical Chemistry and Laboratory Medicine, DGKL-RfB Stiftung Pathobiochemie grant.

Institutional Review Board Statement: Ethics approval was not required as all in vitro samples were anonymized.

Informed Consent Statement: Not applicable.

Data Availability Statement: The data presented in this study are available upon reasonable request from the corresponding author via e-mail.

Acknowledgments: The authors acknowledge the contributions of the technical staff at our institution within this study.

Conflicts of Interest: The authors declare no conflict of interest.

\section{References}

1. Livermore, D.M. Current Epidemiology and Growing Resistance of Gram-Negative Pathogens. Korean J. Intern. Med. 2012, 27, 128-142. [CrossRef]

2. Tängdén, T.; Giske, C.G. Global dissemination of extensively drug-resistant carbapenemase-producing Enterobacteriaceae: Clinical perspectives on detection, treatment and infection control. J. Intern. Med. 2015, 277, 501-512. [CrossRef]

3. Tacconelli, E.; Carrara, E.; Savoldi, A.; Harbarth, S.; Mendelson, M.; Monnet, D.L.; Pulcini, C.; Kahlmeter, G.; Kluytmans, J.; Carmeli, Y.; et al. Discovery, research, and development of new antibiotics: The WHO priority list of antibiotic-resistant bacteria and tuberculosis. Lancet Infect. Dis. 2018, 18, 318-327. [CrossRef]

4. Xu, J.; Murphy, S.; Arias, E.; Kochanek, K. Deaths: Final Data for 2019. Natl. Vital Stat. Rep. 2021, 70. [CrossRef]

5. Hall, M.J.; Levant, S.; DeFrances, C.J. Trends in Inpatient Hospital Deaths: National Hospital Discharge Survey, 2000-2010; No. 118; US Department of Health and Human Services, Centers for Disease Control and Prevention, National Center for Health Statistics: Washington, DC, USA, 2013; pp. 1-8.

6. Liu, V.; Escobar, G.J.; Greene, J.D.; Soule, J.; Whippy, A.; Angus, D.C.; Iwashyna, T.J. Hospital Deaths in Patients With Sepsis From 2 Independent Cohorts. J. Am. Med. Assoc. 2014, 312, 90-92. [CrossRef] [PubMed]

7. Gandra, S.; Trett, A.; Klein, E.Y.; Laxminarayan, R. Is Antimicrobial Resistance a Bigger Problem in Tertiary Care Hospitals Than in Small Community Hospitals in the United States? Clin. Infect. Dis. 2017, 65, 860-863. [CrossRef] [PubMed]

8. WHO. New Report Calls for Urgent Action to Avert Antimicrobial Resistance Crisis. Available online: https://www. who.int/news/item/29-04-2019-new-report-calls-for-urgent-action-to-avert-antimicrobial-resistance-crisis ] (accessed on 25 September 2021).

9. Burnham, J.P.; Olsen, M.A.; Kollef, M.H. Re-estimating annual deaths due to multidrug-resistant organism infections. Infect. Control. Hosp. Epidemiol. 2018, 40, 112-113. [CrossRef]

10. Antimicrobial Threats Report by the Center of Disease Control and Prevention (CDC). 2019. Available online: https://www.cdc. gov/drugresistance/biggest-threats.html (accessed on 25 September 2021).

11. ECDC. Antibiotic Resistance-An Increasing Threat to Human Health. Available online: https://antibiotic.ecdc.europa.eu/en/ publications-data/antibiotic-resistance-increasing-threat-human-health (accessed on 25 September 2021).

12. Cassini, A.; Högberg, L.D.; Plachouras, D.; Quattrocchi, A.; Hoxha, A.; Simonsen, G.S.; Colomb-Cotinat, M.; Kretzschmar, M.E.; Devleesschauwer, B.; Cecchini, M.; et al. Attributable deaths and disability-adjusted life-years caused by infections with antibiotic-resistant bacteria in the EU and the European Economic Area in 2015: A population-level modelling analysis. Lancet Infect. Dis. 2018, 19, 56-66. [CrossRef] 
13. Hackel, M.A.; Tsuji, M.; Yamano, Y.; Echols, R.; Karlowsky, J.A.; Sahm, D.F. In Vitro Activity of the Siderophore Cephalosporin, Cefiderocol, against Carbapenem-Nonsusceptible and Multidrug-Resistant Isolates of Gram-Negative Bacilli Collected Worldwide in 2014 to 2016. Antimicrob. Agents Chemother. 2018, 62, e0196817. [CrossRef]

14. Zhanel, G.G.; Golden, A.R.; Zelenitsky, S.; Wiebe, K.; Lawrence, C.; Adam, H.J.; Idowu, T.; Domalaon, R.; Schweizer, F.; Zhanel, M.A.; et al. Cefiderocol: A Siderophore Cephalosporin with Activity Against Carbapenem-Resistant and Multidrug-Resistant Gram-Negative Bacilli. Drugs 2019, 79, 271-289. [CrossRef]

15. Kazmierczak, K.M.; Tsuji, M.; Wise, M.G.; Hackel, M.; Yamano, Y.; Echols, R.; Sahm, D.F. In vitro activity of cefiderocol, a siderophore cephalosporin, against a recent collection of clinically relevant carbapenem-non-susceptible Gram-negative bacilli, including serine carbapenemase- and metallo- $\beta$-lactamase-producing isolates (SIDERO-WT-2014 Study). Int. J. Antimicrob. Agents 2019, 53, 177-184. [CrossRef]

16. Sato, T.; Yamawaki, K. Cefiderocol: Discovery, Chemistry, and In Vivo Profiles of a Novel Siderophore Cephalosporin. Clin. Infect. Dis. 2019, 69, S538-S543. [CrossRef]

17. Naas, T.; Lina, G.; Santerre Henriksen, A.; Longshaw, C.; Jehl, F. In vitro activity of cefiderocol and comparators against isolates of Gram-negative pathogens from a range of infection sources: SIDERO-WT-2014-2018 studies in France. JAC Antimicrob. Resist. 2021, 3, dlab081. [CrossRef]

18. Mushtaq, S.; Sadouki, Z.; Vickers, A.; Livermore, D.M.; Woodford, N. In Vitro Activity of Cefiderocol, a Siderophore Cephalosporin, against Multidrug-Resistant Gram-Negative Bacteria. Antimicrob Agents Chemother. 2020, 64, e01582-20. [CrossRef] [PubMed]

19. Malik, S.; Kaminski, M.; Landman, D.; Quale, J. Cefiderocol Resistance in Acinetobacter baumannii: Roles of $\beta$-Lactamases, Siderophore Receptors, and Penicillin Binding Protein 3. Antimicrob Agents Chemother. 2020, 64, e0122120. [CrossRef] [PubMed]

20. Iregui, A.; Khan, Z.; Landman, D.; Quale, J. Activity of Cefiderocol Against Enterobacterales, Pseudomonas aeruginosa, and Acinetobacter baumannii Endemic to Medical Centers in New York City. Microb. Drug Resist. 2020, 26, 722-726. [CrossRef] [PubMed]

21. Poirel, L.; Sadek, M.; Nordmann, P. Contribution of PER-type and NDM-type B-lactamases to cefiderocol resistance in Acinetobacter baumannii. Antimicrob Agents Chemother. 2021, 65, e0087721. [CrossRef] [PubMed]

22. Jacobs, M.R.; Abdelhamed, A.M.; Good, C.E.; Rhoads, D.D.; Hujer, A.M.; Domitrovic, T.N.; Rudin, S.D.; Richter, S.S.; van Duin, D.; Kreiswirth, B.N. ARGONAUT-I: Activity of cefiderocol (S-649266), a siderophore cephalosporin, against Gram-negative bacteria, including carbapenem-resistant nonfermenters and Enterobacteriaceae with defined extended-spectrum $\beta$-lactamases and carbapenemases. Antimicrob. Agents Chemother. 2019, 63, e0180118. [CrossRef]

23. Ito, A.; Nishikawa, T.; Matsumoto, S.; Yoshizawa, H.; Sato, T.; Nakamura, R.; Tsuji, M.; Yamano, Y. Siderophore Cephalosporin Cefiderocol Utilizes Ferric Iron Transporter Systems for Antibacterial Activity against Pseudomonas aeruginosa. Antimicrob. Agents Chemother. 2016, 60, 7396-7401. [CrossRef]

24. Ito-Horiyama, T.; Ishii, Y.; Ito, A.; Sato, T.; Nakamura, R.; Fukuhara, N.; Tsuji, M.; Yamano, Y.; Yamaguchi, K.; Tateda, K. Stability of Novel Siderophore Cephalosporin S-649266 against Clinically Relevant Carbapenemases. Antimicrob. Agents Chemother. 2016, 60, 4384-4386. [CrossRef] [PubMed]

25. Poirel, L.; Kieffer, N.; Nordmann, P. Stability of cefiderocol against clinically significant broad-spectrum oxacillinases. Int. J. Antimicrob. Agents 2018, 52, 866-867. [CrossRef]

26. Smiljanic, M.; Kaase, M.; Ahmad-Nejad, P.; Ghebremedhin, B. Comparison of in-house and commercial real time-PCR based carbapenemase gene detection methods in Enterobacteriaceae and non-fermenting gram-negative bacterial isolates. Ann. Clin. Microbiol. Antimicrob. 2017, 16,1-7. [CrossRef]

27. Pazzini, C.; Ahmad-Nejad, P.; Ghebremedhin, B. Ceftolozane/tazobactam susceptibility testing in extended-spectrum betalactamase- and carbapenemase-producing gram-negative bacteria of various clonal lineages. Eur. J. Microbiol. Immunol. 2019, 9, 1-4. [CrossRef]

28. Ghebremedhin, B.; Halstenbach, A.; Smiljanić, M.; Kaase, M.; Ahmad-Nejad, P. MALDI-TOF MS based carbapenemase detection from culture isolates and from positive blood culture vials. Ann. Clin. Microbiol. Antimicrob. 2016, 15, 5. [CrossRef] [PubMed]

29. Woodford, N.; Ellington, M.J.; Coelho, J.M.; Turton, J.; Ward, M.E.; Brown, S.; Amyes, S.G.; Livermore, D.M. Multiplex PCR for genes encoding prevalent OXA carbapenemases in Acinetobacter spp. Int. J. Antimicrob. Agents 2006, 27, 351-353. [CrossRef] [PubMed]

30. Kresken, M.; Körber-Irrgang, B.; Korte-Berwanger, M.; Pfennigwerth, N.; Gatermann, S.G.; Seifert, H. German Carbapenem Resistance Study Group Dissemination of carbapenem-resistant Pseudomonas aeruginosa isolates and their susceptibilities to ceftolozane-tazobactam in Germany. Int. J. Antimicrob. Agents 2020, 55, 105959. [CrossRef] [PubMed]

31. The European Committee on Antimicrobial Susceptibility Testing (EUCAST). Breakpoint Tables for Interpretation of Mics and Zone Diameters, (Version 11.0). The European Committee on Antimicrobial Susceptibility Testing. 2021. Available online: https://www.eucast.org/fileadmin/src/media/PDFs/EUCAST_files/Breakpoint_tables/v_11.0_Breakpoint_Tables.pdf (accessed on 31 August 2021). 